\title{
Dynamics of crystallization and dissolution of calcium orthophosphates at the near-molecular level
}

\author{
WANG LiJun $^{1^{*}}$, LU JianWei $^{1}, \mathrm{XU}_{\text {FangSen }}{ }^{1} \&$ ZHANG FuSuo ${ }^{2 *}$ \\ ${ }^{1}$ College of Resources and Environment, Huazhong Agricultural University, Wuhan 430070, China; \\ ${ }^{2}$ College of Resources and Environmental Sciences, China Agricultural University, Beijing 100193, China
}

Received April 27, 2010; Accepted August 26, 2010

\begin{abstract}
Although extensive investigations of biogenic and geological calcium phosphate crystallization/dissolution and their phase transformations have been performed, the mechanisms of crystallization and dissolution of sparingly soluble calcium phosphates in geological settings have not been completely determined at the near-molecular level. In particular, an understanding of the physical-chemical processes at the earliest nucleation stage and the subsequent crystal surface dynamics in soil solutions is lacking. This review focuses on the earliest events in homo/heterogeneous nucleation from an initial supersaturated solution phase, the subsequent growth of calcium phosphate phases, and the relevant influences of the presence of additional inorganic and organic molecules in both geological and biological settings. These studies have implications for the understanding of the complex processes of calcium phosphate transformations in soils, and provide possible physical-chemical mechanisms for the biogeochemical behavior of phosphorus at the near-molecular level.
\end{abstract}

calcium orthophosphates, crystallization, dissolution, AFM

Citation: Wang L J, Lu J W, Xu F S, et al. Dynamics of crystallization and dissolution of calcium orthophosphates at the near-molecular level. Chinese Sci Bull, 2011, 56: 713-721, doi: 10.1007/s11434-010-4184-2

After nitrogen, phosphorus $(\mathrm{P})$ is the main limiting macronutrient for plant growth. It is present in only minute quantities in the Earth's crust $(0.09 \mathrm{wt} \%)$ [1]. The US Geological Survey (USGS) estimates that around 62 billion tonnes of phosphate remain in the ground. This includes 15 billion tonnes of deposits that are mineable at present [2]. The demand for $\mathrm{P}$ fertilizers is predicted to grow by $2.5 \%-3 \%$ per year for the next 5 years. If this rate continues, the world's reserves would last for around 125 years [2]. Apatite $\left[\mathrm{Ca}_{10}\left(\mathrm{PO}_{4}\right)_{6}(\mathrm{OH}, \mathrm{F}, \mathrm{Cl})_{2}\right]$ is the primary inorganic source of $\mathrm{P}$ in phosphate-bearing minerals. Plant roots and microbes, including fungi and bacteria, are able to solubilize $\mathrm{P}$ directly from minerals such as apatite $[3,4]$, especially plants that are known to increase plant-available $\mathrm{P}$ by promoting dissolution of various phosphate minerals [5]. However, free $\mathrm{P}$ released in soils by dissolution of sparingly soluble $\mathrm{P}$ is

*Corresponding authors (email: ljwang@ mail.hzau.edu.cn; zhangfs@cau.edu.cn) rapidly immobilized by sorption onto minerals or hydrous oxides of $\mathrm{Al}$ and $\mathrm{Fe}$ or onto extracellular matrices (ECM) of microbes to form various phosphate phases. During the competing processes of mineral dissolution and precipitation, various components, structures, crystal phases, and hydration states of phosphates are present in soil solutions or on soil solid surfaces, and calcium orthophosphates $(\mathrm{CaP})$ are the main mineral constituents among the biogenic and geological calcium phosphate minerals.

The crystallization of CaPs involves the formation of metastable intermediate precursor phases followed by transformation to the final product. These intermediate phases include an initial amorphous $\mathrm{CaP}$ phase (ACP), brushite $\left(\mathrm{CaHPO}_{4} \cdot 2 \mathrm{H}_{2} \mathrm{O}\right.$, DCPD, monoclinic Ia), octacalcium phosphate $\left(\mathrm{Ca}_{8}\left(\mathrm{HPO}_{4}\right)_{2}\left(\mathrm{PO}_{4}\right)_{4} \cdot 5 \mathrm{H}_{2} \mathrm{O}\right.$, OCP, triclinic $P \overline{\mathbf{1}})$, tricalcium phosphate $\left(\alpha(\beta)-\mathrm{Ca}_{3}\left(\mathrm{PO}_{4}\right)_{2}, \quad \alpha(\beta)-\mathrm{TCP}\right.$, monoclinic (rhombohedral) $P 2_{1} / a(R 3 C h)$ ), and, the least soluble, hydroxyapatite $\left(\mathrm{Ca}_{10}\left(\mathrm{PO}_{4}\right)_{6}(\mathrm{OH})_{2}\right.$, HAP, hexagonal 
$\left.\mathrm{P}_{3} / m\right)$ as the final stable phase. Most CaPs are sparingly soluble in water, but all dissolve in acids. The calcium to phosphate molar ratios $(\mathrm{Ca} / \mathrm{P})$ and the solubilities are important parameters in distinguishing between the phases. In general, the lower the $\mathrm{Ca} / \mathrm{P}$ ratio, the more acidic and soluble the $\mathrm{CaP}$ phase [6]. Various metastable precursor phases subsequently dissolve as the precipitation reactions proceed [7]. Soil organic components, including organic acids, polysaccharides, proteins and enzymes, excreted by plant roots and microbes may exert different influences on crystallization or dissolution. The $\mathrm{pH}$ and the presence in soil solutions of small inorganic ions such as $\mathrm{Mg}^{2+}, \mathrm{Fe}^{2+} / \mathrm{Fe}^{3+}$, and $\mathrm{Al}^{3+}$ will also influence the lifetime of metastable precursor phases formed during the dissolution-recrystallization process. In addition, it becomes more difficult to predict $\mathrm{CaP}$ phases in soils if the incorporation or substitution of foreign inorganic ions in the crystal lattice, or their adsorption onto crystal faces, takes place.

To understand these complicated processes, the dynamics of the crystallization and dissolution of $\mathrm{CaPs}$ will be analyzed quantitatively. We will discuss some important parameters related to crystal nucleation and growth/dissolution, including supersaturation/undersaturation, $\mathrm{pH}$, ionic strength, and the ratio of calcium to phosphate activities. We will then focus on the dynamics of crystallization/dissolution, by a combination of bulk and surface crystallization, in the presence of various organic or inorganic species pertinent to geological $\mathrm{CaP}$ minerals. This will provide insights into the physical mechanisms of crystal growth/dissolution and phase stability, and into the influences of proteins, peptides, or other small molecules. Deciphering these microscopic surface processes and mechanisms will be helpful in understanding $\mathrm{P}$ behaviors in soils and enhancing $\mathrm{P}$ availability.

\section{Nucleation and growth of calcium orthophosphate crystals from solutions}

Solution properties such as supersaturation, $\mathrm{pH}$, ionic strength $(I)$, and the ratio of cations to anions determine the thermodynamic relationship between the solution and the crystal; these parameters can also influence kinetics. In phosphate systems, a shift to lower $\mathrm{pH}$ will typically change the saturation state and change the balance of phosphate species from $\mathrm{PO}_{4}{ }^{3-}$ to $\mathrm{HPO}_{4}{ }^{2-}$ to $\mathrm{H}_{2} \mathrm{PO}_{4}^{-}$. At the mineral surface, the $\mathrm{pH}$ can shift the surface charge by changing the distribution of proton and hydroxyl groups hydrating the interface. This may change the activation barriers associated with crystallization, as the hydrating ions must be removed (desolvation) to allow a crystallizing ion to attach to the crystal surface. The ionic strength influences electrostatic interactions between ions in solution or electrostatic interactions between ions in solution and on the mineral surface. The ionic strength is defined as $I=1 / 2 \sum_{i} c_{i} z_{i}^{2}\left(c_{i}\right.$ and $z_{i}$ are the concentration and the charge, respectively, of the $i$ th ion). Interactions between ions in electrolyte solutions reduce the effective free ion concentrations from their stoichiometric values: $a_{i}=c_{i} y_{ \pm}\left(a_{i}\right.$ is the activity, $y_{ \pm}$is the mean activity coefficient). Using an extended form of the Debye-Hückel equation, such as that proposed by Davies, $y_{ \pm}$can be calculated as

$$
\log y_{ \pm}=-A z_{i}^{2}\left(\frac{\sqrt{I}}{1+\sqrt{I}}-0.3 I\right)
$$

$A$ is a temperature-dependent constant. Another important thermodynamic parameter for driving $\mathrm{CaP}$ crystallization is the supersaturation, $S$, defined as

$$
\ln S=\frac{\Delta \mu}{k T},
$$

$k$ is the Boltzmann constant, $T$ is the absolute temperature. The direct definition is the ratio of the actual ion activity product (IAP) and its value at equilibrium $\left(K_{\mathrm{sp}}\right.$, the solubility product for a precipitation reaction):

$$
S=\left(\frac{\prod_{i} a_{i}^{m_{i}}}{K_{\mathrm{sp}}}\right)^{1 / n},
$$

$n$ is the number of growth units per molecule. For example, $\mathrm{CaHPO}_{4} \cdot 2 \mathrm{H}_{2} \mathrm{O}$ (DCPD), $n=2$ (excluding hydrate water); $\mathrm{Ca}_{5}\left(\mathrm{PO}_{4}\right)_{3}(\mathrm{OH})(1 / 2 \mathrm{HAP}), n=9$, corresponding $S_{\mathrm{HAP}}=$ $\left(\left[a\left(\mathrm{Ca}^{2+}\right)\right]^{5}\left[a\left(\mathrm{PO}_{4}^{3-}\right)\right]^{3}[a(\mathrm{OH})]^{1 /} / K_{\text {sp }, \mathrm{HAP}}\right)^{1 / 9}$. The activation barriers for the cation and anion may not be the same. The cation to anion activity ratio $(\mathrm{Ca} / \mathrm{P})$ will directly influence the growth rates of crystallization, which will depend on the concentration of the rate-limiting ion rather than simply on supersaturation [8-10]. The growth of a multicomponent HAP crystal relies on the relative rates of adsorption and desorption of the various ions or growth units and their desolvation processes [11].

In general, the phase stability and crystal lattice substitutions of $\mathrm{CaPs}$ must take into account the probable influence of extraneous small ions such as $\mathrm{Mg}^{2+}, \mathrm{Zn}^{2+}, \mathrm{CO}_{3}{ }^{2-}$, and $\mathrm{SO}_{4}{ }^{2-}$ in soil solutions. Observation of deposits of ACP indicates that the presence of $\mathrm{Mg}^{2+}$ is able to stabilize this phase. A very recent study showed that although the short-range structure around the $\mathrm{Mg}^{2+}$ ion is different in the various minerals studied, they all involve a shortening of the $\mathrm{Mg}-\mathrm{O}$ bond length, and the compact structure around magnesium introduces distortion in the host mineral, thus inhibiting its crystallization [12]. The apparent stabilization of ACP may be because of the binding of $\mathrm{Mg}^{2+}$ to newly formed nuclei, not only preventing these from reaching a critical growth size and subsequent Ostwald maturation, but also leading to redissolution [13]. The equilibrium adsorption of $\mathrm{Zn}^{2+}$ on HAP suggests that the formation of zinc phosphate (hopeite) dominates the surface properties and 
determines the degree of inhibition of HAP crystal growth [14]. At neutral $\mathrm{pH}$, anionic organic macromolecules such as proteins show a considerably greater influence on HAP crystallization than do positive or neutral species, suggesting that they bind to the CaP nanoclusters; this is especially the case for proteins with a hydrophilic carboxylate head that can significantly shorten the induction time of OCP [15] or HAP [16,17], and promote the formation of the corresponding crystals. Small amino acids can also promote rapid transformation of amorphous CaPs into apatite [18]. However, some aspartic-acid-rich proteins and peptides could significantly inhibit the formation of calcium-based minerals [19-23]. Certain polyelectrolytes induced HAP crystallization at low temperatures and inhibited its nucleation at high temperatures [24].

Phase transformations in crystallizing $\mathrm{CaPs}$ is an important point, and more acidic $\mathrm{CaP}$ phases are involved in this process that later transform to HAP. Many studies have shown that biological control, either by localizing crystallization or by stabilizing the otherwise short-lived amorphous forms, could be attributed to proteins and their cooperative combinations, peptides and inorganic ions, on the assumption that each exhibits roughly the same influence in inhibiting the nucleation and growth of crystallites [6,7]. A very recent result showed that citrate can regulate two interfaces in opposite ways, which decreases and increases the stabilities of brushite and hydroxyapatite surfaces, respectively, in the solution. Thus, the interfacial energy barrier can be greatly reduced in the presence of citrate and the transformation from DCPD to HAP is induced [25]. In general, DCPD is considered to be a precursor phase of OCP and HAP, and it is transformed into HAP by a dissolutionrecrystallization mechanism [26]. During this transformation, some organic macromolecules retard phase transformation by inhibiting the dissolution process $[6,7]$.

In the presence of foreign objects such as soil particles or cell-wall surfaces of soil bacteria, the nucleation barrier may be reduced to: $\Delta G_{\text {hetero }}=\Delta G_{\text {homo }} f(m, x)(0 \leqslant f(m, x) \leqslant 1)$. The height of the homogeneous nucleation in a supersaturated solution is defined as $\Delta G_{\mathrm{homo}}=16 \pi \lambda_{\mathrm{cf}}^{3} \omega^{2} / 3|\Delta \mu|^{2}(\omega$ is the volume of the growth unit, $\gamma_{\mathrm{cf}}$ is the surface free energy of the solid-liquid interface). The interfacial correlation factor $f(m, x)$ describes the lowering of the nucleation barrier as a result of the presence of foreign objects, and will increase with supersaturation [27]. The interfacial interaction parameter is defined as $m=\left(\gamma_{\mathrm{sf}}-\gamma_{\mathrm{sc}}\right) / \gamma_{\mathrm{cf}}\left(\gamma_{\mathrm{sf}}, \gamma_{\mathrm{sc}}\right.$ and $\gamma_{\mathrm{cf}}$ are the interfacial free energies between the solid particle (s), the fluid (f), and the crystal nucleus (c) interface); the size of the solid particles is $x=R^{\mathrm{s}} / r_{\mathrm{c}}\left(R^{\mathrm{s}}\right.$ is the average radius of spherical particles), and $r_{\mathrm{c}}$ is the critical size of nuclei defined as $\left(r_{\mathrm{c}}=2 \varpi \lambda_{\mathrm{cf}} / k T \operatorname{In} S\right)$. When the interaction between the nucleating phase and the solid surface is optimal, $f(m, x) \rightarrow 0$; conversely, if the interfacial correlation is very poor, $f(m, x) \rightarrow 1$, and the surface or the substrate exerts almost no influence on the CaP nucleation barrier [27].

\section{Crystal growth and dissolution at the near- molecular level}

\subsection{Molecular mechanisms of crystal surface growth}

In the late 1990s, two research groups in the United States and Germany used in situ atomic force microscopy (AFM) coupled with a fluid cell to investigate the kinetics and thermodynamics of the crystal growth of proteins, calcite, and barite [28-30]. AFM has emerged as an important investigative tool because its features make it uniquely suitable for applying well-founded molecular-scale concepts of crystallization to the analysis of results. Quantitative AFM studies enable incisive investigations of the effects of modulators on the morphology, kinetics, and energetics of crystal nucleation and growth, including the velocity of atomic steps and the critical length for step motion versus supersaturation in a wide variety of solution-grown crystals $[28,29]$.

Crystal surfaces consist of flat regions called terraces, and raised partial layers called steps (Figure 1). The driving force for growth of a crystal surface from solution is the extent to which solute concentrations exceed the levels at equilibrium, i.e., supersaturation. Growth from a supersaturated solution occurs because the flux of molecules attaching to the crystal surface exceeds the flux of molecules detaching from the surface. The probability that a molecule will detach from the crystal is solely determined by the strength of its bonds to its neighbors. A molecule is able to make more bonds to neighboring molecules if it attaches to a complementary step edge than if it lands on a flat terrace [30,31]. Thus, permanent attachment occurs almost exclusively at step edges. Kink sites are the most reactive portions of step edges (Figure 1(A)). Because molecules at the step edge have fewer bonds to break than molecules embedded in the crystal lattice, nearly all detachment also occurs at step edges. Nearly all crystals contain dislocations and defects, i.e., breaks in the crystal lattice that create steps on the surface that will support crystal growth (Figure 1(B)). However, if there are no pre-existing steps, nucleation of two-dimensional islands is an alternative way of forming new steps (Figure 1(A)) [30,31]. Figure 1(B) shows the geometry of a dislocation hillock. The existence of a critical size leads to the formation of a spiral structure because the new segment of the step cannot move until it reaches that size. Because attachment and detachment are statistically independent, the rate of step advancement, $v$, exhibits linear kinetics, i.e., it is linearly proportional to the difference between the actual and equilibrium concentrations of the solute, $C-C_{\mathrm{e}}$, according to $v=\beta \omega\left(C-C_{\mathrm{e}}\right)(\beta$ is the kinetic coefficient). Chernov [31] derived the common expression $v=\beta \omega K_{\mathrm{sp}}\left(S^{1 / n}-1\right)$. Besides the step speed, the characteristics of the growth spiral are the distance between steps (i.e., terrace width) and shape. The terrace width, $W$, is important in determining the growth rate, $R$. As Figure 1(B) 


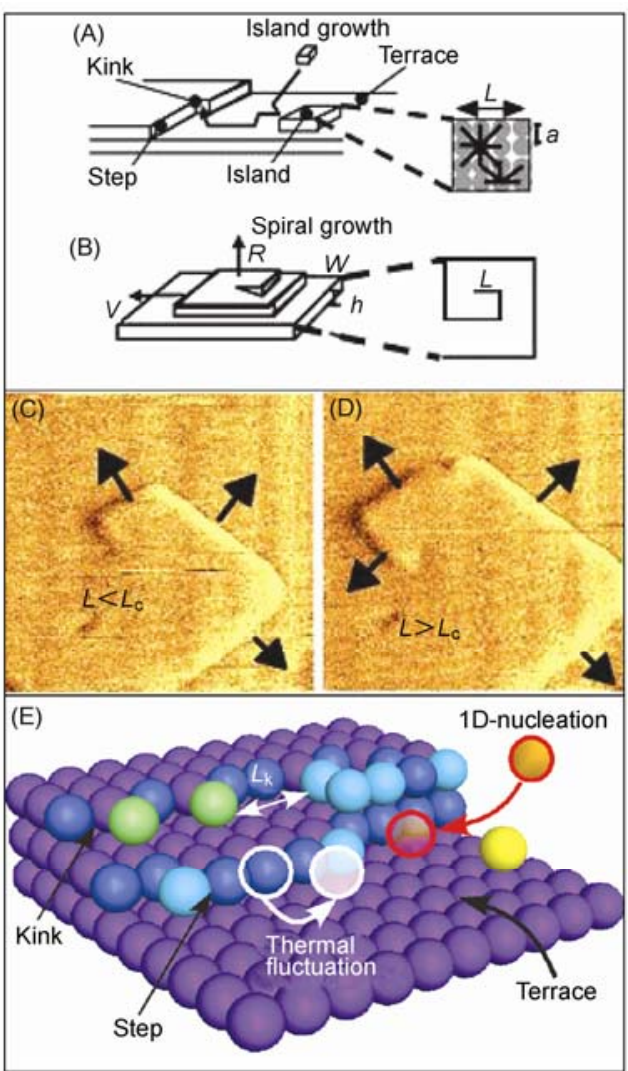

Figure 1 Illustration of crystal growth by attachment of molecules to step edges on (A) islands or (B) dislocation spirals. (C), (D) Schematic top view of growth spiral with $L<L_{\mathrm{c}}$ at $t=0$, and $L>L_{\mathrm{c}}$ at a later time. Step edges with arrows are advancing [35]. (E) Schematic illustrating terrace, step, and kinks on crystal surface. Kinks are created either via movements of molecules on the step edge (thermal fluctuations) or attachment of new solute molecules from solution (1D nucleation). The step advances because addition of molecules to right- and left-facing kinks leads to their lateral movement and eventual annihilation [32].

shows, $R$ is equal to the hillock slope, $p$, times the step speed, $v$. But the hillock slope is just $h / W$, where $h$ is the step height, which is fixed by the crystal structure. Controls on terrace width are therefore also important in determining growth rates [32].

Kinks are created either by movements of molecules on the step edge (thermal fluctuations) or by attachment of new solute molecules from solution (1D nucleation). The step advances because addition of molecules to right- and left-facing kinks leads to their lateral movement and eventual annihilation (Figure 1(E)). At equilibrium, the average spacing between kinks is $L_{\mathrm{K}} \propto(b / 2) \exp (\varepsilon / k T)$ ( $b$ is the lattice spacing). For sparingly soluble CaPs, the density of kink sites and the energy barriers at equilibrium are the major determinants of the kinetics of the attachment/detachment process at step edges [32]. In general, the barrier to desolvation, i.e., the breaking of bonds to solvent molecules, appears to be the dominant barrier to attachment of a solute molecule to the step. When detaching from a step, the primary barriers are the bonds to adjacent molecules in the crystal. The speed of step movement reflects these barriers. As the solute concentration (and resultant flux of solute to the step) is increased, the step speed increases. The kinetic coefficient $(\beta)$ is the slope of this relationship. Determining the dependence of $\beta$ on temperature allows calculation of the energy barriers that ultimately control the growth kinetics [28]. Thus, measuring its changes in the presence of inorganic or organic additive molecules provides a quantitative assessment of the molecular mechanisms underlying changes in growth kinetics [33,34].

Many AFM studies have investigated step velocity and morphology [35]. A basic parameter controlling step growth is the step length; the classic Gibbs-Thomson (G-T) effect predicts that the critical length $\left(L_{\mathrm{c}}\right)$ near equilibrium is related to the step-edge free energy $(\gamma)$ and supersaturation:

$$
L_{\mathrm{c}}=\frac{\omega \gamma}{\Delta \mu}=\frac{\varpi \gamma}{k T \ln S} .
$$

The notion of critical size $L_{\mathrm{c}}$ is a crucial concept for understanding the probability of nucleation of islands, the rate of crystal growth, and the morphology of a growing crystal surface. The change in chemical energy per molecule that occurs as molecules leave a solution and enter the solid state is expressed relative to that of the bulk crystal. Because systems always move towards a state of minimum free energy, growth occurs when the change in chemical potential is negative, and dissolution occurs when it is positive [35]. Compared to molecules within the crystal, the molecules that comprise the surface have a relative excess of free energy, called the surface energy. Similarly, new steps created on a surface (as by birth of an island) have an excess free energy known as the step-edge energy. Free energy changes during step growth therefore reflect two terms: (1) a stabilizing term because of the drop in chemical potential per attaching molecule; and (2) a destabilizing term because of an increase in edge free energy [35]. The first term is negative and proportional to the number of molecules in the island, and the second term is positive and proportional to the length of the island perimeter [35]. The difference between these changes goes to a maximum at the critical radius. Islands smaller than the critical radius decrease free energy by shrinking, and those larger than the critical radius decrease free energy by growing. Thus, smaller islands dissolve and larger ones grow [29,32]. The relationship between supersaturation and the critical radius of an island is expressed by the G-T relationship. Experimental determination of this relationship allows extraction of the step-edge energy, the most important thermodynamic factor controlling growth from solutions [29,32]. This concept also applies to step segments. A step segment advances as a new step edge is created adjacent to the segment (Figure 1(C) and (D)).

The critical length will vary depending on the step orientation and geometry of the crystal. For the example of DCPD (Figure 2(A)), the critical length of the $i$ th step is $L_{\mathrm{c}, \mathrm{i}}=b_{\mathrm{i}} h\left(\gamma_{1} b_{1}+\gamma_{2} b_{2}+\gamma_{3} b_{3}\right) / \Delta \mu$, thus the ratios of $L_{\mathrm{c} 1} / L_{\mathrm{c} 2} / L_{\mathrm{c} 3}$ are expected to be $b_{1} / b_{2} / b_{3}$. When two steps $[10 \overline{1}]_{\mathrm{Cc}}$ and 
(A)

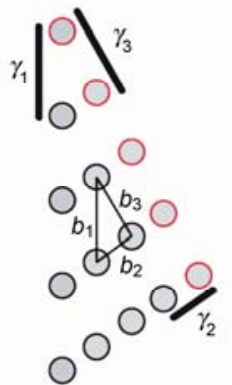

(B)

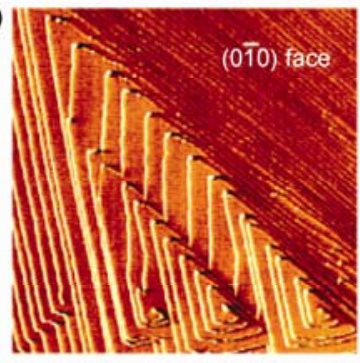

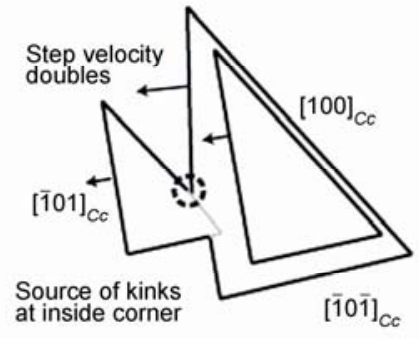

Figure 2 (A) The relationship between critical length and step-edge free energies depends on the geometry of the crystal. Schematic of the addition of a row of atoms (in red) to an existing step for triangular geometry (such as brushite). Dark lines indicate the newly created edge length, each with its own step-edge free energy, $\gamma_{\text {edge }}\left(\mathrm{J} \mathrm{m}^{-1}\right)$ [37]. (B) AFM image $(6 \mu \mathrm{m} \times 6 \mu \mathrm{m})$ of multiple merging hillocks on a growing brushite surface. The velocity of the [101] step direction (noted with arrows in the schematic) depends upon the corner type and roughly doubles when it terminates at an inside rather than an outside corner [37].

$[\overline{100}]_{\mathrm{Cc}}$ meet, they form an inside angle where there is no potential barrier for kink generation. This angle can therefore serve as a kink source that can accelerate $[10 \overline{1}]_{\mathrm{Cc}}$ step propagation when motion is limited by kink nucleation (Figure 2(B)) [36,37].

\subsection{Dissolution model of sparingly soluble calcium orthophosphates at the nanoscale}

The constant composition technique for measuring bulk DCPD dissolution rates showed that the dissolution rate slowed almost to a halt before all the starting seed crystals were consumed, despite a constant degree of undersaturation [37]. This finding is contrary to the Ostwald-Freundlich description of solubility, which expresses solubility as a function of interfacial tension and particle size, and predicts that the solubility increases as particle size is reduced. More commonly, the solubility is equated with the solubility product $K_{\mathrm{sp}}$ and considered to be independent of surface effects [37]. To gain a clearer understanding of this unusual phenomenon, Tang and Orme et al. employed in situ AFM to directly monitor the DCPD surface, and found that the surface energy is important during dissolution [38,39]. What is immediately clear from these dissolution sequences is that not all steps are freely dissolving, despite the undersaturated conditions. Rather, morphology, size, and orientation all have a role. Islands dissolve readily but pits dissolve comparatively slowly.

From an energetic perspective, dissolution is guided by the incremental changes in Gibbs free energy because of the removal of a row of atoms from a step. This involves both the chemical potential and surface energy terms. The degree to which step free energies limit dissolution depends on the geometry of the surface. A surface with some roughness because of islands, hillocks, and/or steps will undergo spontaneous dissolution because both the chemical energy term and the step free energy term are negative because of the simultaneous removal of atoms from the solid and the reduction of step length. In this case, there is no critical size: the dissolution of all steps is independent of their length [37]. However, some existing pits, and pits emanating from dislocations, may or may not dissolve spontaneously, depending on the step lengths. As a step dissolves, its length increases. Because these step edges increase the energy of the system, this is an energetically unfavorable event until the step lengths are sufficiently large for the energy decrease associated with dissolution to overcome the energy increase associated with creating more edge length. This is completely analogous to the discussions of a critical length for step motion during growth [37]. For DCPD, which has a triangular symmetry, the incremental change in, the Gibbs free energy is $\Delta G_{i} / \Delta x_{i}=-\left(L / b_{i}\right)|\Delta \mu|+h \sum_{i} \gamma_{i} b_{i}$

$L$ is the length of the dissolving step, $b_{\mathrm{i}}$ and $\gamma_{\mathrm{i}}$ are the crystal spacing and step free energy in the $i$ th direction, respectively [37]. Generally, the step free energy term is constant; thus, once the step length is sufficiently large or the solution is sufficiently undersaturated, the negative chemical potential term wins and dissolution becomes energetically favorable. At the farthest extreme, on a defect-free flat surface, a pit must be nucleated, which also involves the creation of step edges. In this instance, the removal of one molecule must overcome the free energy term, $\Delta G / \Delta n=$ $-|\Delta \mu|+h \sum_{i} \gamma_{i} b_{i}$ [37].

Near equilibrium, sparingly soluble CaPs with high surface energies or low solubility [6] will have dissolution rates that are moderated by their surface geometry and size. The effects become more important as the particle size approaches the critical length because more of the surface is likely to be composed of steps shorter than the critical length. This new dissolution model incorporating particle size considerations [38,39] can explain bulk demineralization of sparingly soluble salts such as HAP [40-44], which is generally initiated and accompanied by the formation and development of pits on the crystal surfaces; the dissolution rates are also determined by the pit densities and spreading velocities. The rate of step movement from a pit of radius $r$ 
can be obtained from:

$$
\begin{gathered}
R(r)=R_{\infty}\left[1-\frac{e^{(1-S) r^{*} / r}-1}{e^{1-S}-1}\right] \\
\text { or } R(r) \approx R_{\infty}\left(1-\frac{r^{*}}{r}\right),
\end{gathered}
$$

where $r^{*}$ is the critical radius for the formation of a two-dimensional pit/dissolution step, $S$ is the degree of undersaturation, and $R_{\infty}$ is the velocity of dissolution steps at $r \rightarrow \infty$. According to classical nucleation theory (CNT), the free energy, $\Delta G$, for the nucleation of a pit is:

$$
\Delta G(r)=\frac{\pi r^{2} h}{\varpi} k T \ln S+2 \pi r h \gamma,
$$

where $h$ is the pit depth, and $\gamma$ is the surface free energy. When $S<1$, the solutions are undersaturated, and the first term on the right-hand side of equation (3) is negative, reflecting the decrease in chemical potential, but the second term is positive because of the increase in surface roughness (pit formation) with loss of crystal mass and expansion of the solid-liquid interfacial area. Thus, the dependence of the Gibbs free energy on the radius $(r)$ passes through a maximum, and the critical size (i.e., $r^{*}$ ) can be obtained by setting $\partial(\Delta G) / \partial(r)=0$, thus:

$$
r^{*}=\frac{\gamma \varpi}{|k T \ln S|}
$$

Eq. (2) shows that only pits which are larger than $r^{*}$ provide active dissolution sites that contribute to dissolution. When $r$ is closer to $r^{*}$, there is no fast movement of its stepwave and the dissolution rate approaches zero. The application of this model demonstrates that for $\mathrm{CaP}$ crystallite sizes approaching the critical values, the bulk rates decrease markedly with time, despite the sustained driving force $[38,42]$.

\section{Crystallization and dissolution modulation of calcium orthophosphates through impurity interactions at the near-molecular level}

Four mechanisms have been proposed for crystallization modulation through impurity (including organic and inorganic molecules) interactions: (1) step pinning [45], (2) incorporation [34], (3) kink blocking, and (4) step-edge adsorption. Each of these major mechanisms for growth inhibition exhibits a characteristic dependence of step speed on supersaturation and impurity concentration [35]. A new mechanism has been proposed for the interaction of citrate with DCPD in which impurity molecules reduce step density without influencing the step speed and morphology [46]. This explains the corresponding inhibition of the bulk growth rate of DCPD seeds. Taking the average distance between two steps in a spiral growth as $\lambda_{0}$ (spacing) and their heights as $d_{h k l}$, the growth rate of one face is given by $R_{\mathrm{hkl}}=v_{\text {step }} d_{\mathrm{hkl}} / \lambda_{0}$, showing that a lower density (larger spacing between parallel steps) has a lower face growth rate. The step density on spiral growth hillocks is related to the critical length $L_{\mathrm{c}}$ for step propagation, and, in particular, a larger terrace width (as was observed in the presence of citrate) corresponds to a larger critical length. With the supersaturation kept constant, the critical length $\left(L_{\mathrm{c}} \propto \gamma / \Delta \mu\right)$ is proportional to the step free energy. Thus, the larger terrace widths can be explained by an increased step free energy. A thin layer wicking method was used to measure the surface energies of DCPD powders in the absence and presence of citrate and showed that the interfacial energy increased by $95 \%$ at $10 \mu \mathrm{mol} / \mathrm{L}$ citrate [46]. In the light of the foregoing results, it is appropriate to apply these surface analysis methods to $\mathrm{CaP}$ minerals such as HAP in soils. Surface growth and dissolution rates can be quantitatively measured in the presence of various molecules which may be present in soil solutions following their adsorption onto HAP (100) surfaces or steps (Figure 3(A) and (B)), or onto the pits of a DCPD (010) face (Figure 3(C) and (D)). This directly connects microscopic processes to macroscopic transformations of rhizosphere $P$.

The dissolution process in the presence of inorganic acids such as $\mathrm{HCl}$ is simpler than it is in the presence of organic acids, including citrate, oxalates and aspartic acids, because each of these organic acids interacts with calcium via carboxyl groups (calcium chelation) in different geometries. A model of a (100) hydroxyapatite (HAP) face and citrate reveals experimentally that only a side carboxylate and a surface calcium ion are involved in the binding effect during citrate adsorption, which is contrary to previous results using Langmuir adsorption and computer simulations [47]. Furthermore, the adsorbed citrate molecules can use their free carboxylate and hydroxyl groups to self-assemble on the HAP surface via intermolecular hydrogen bonding

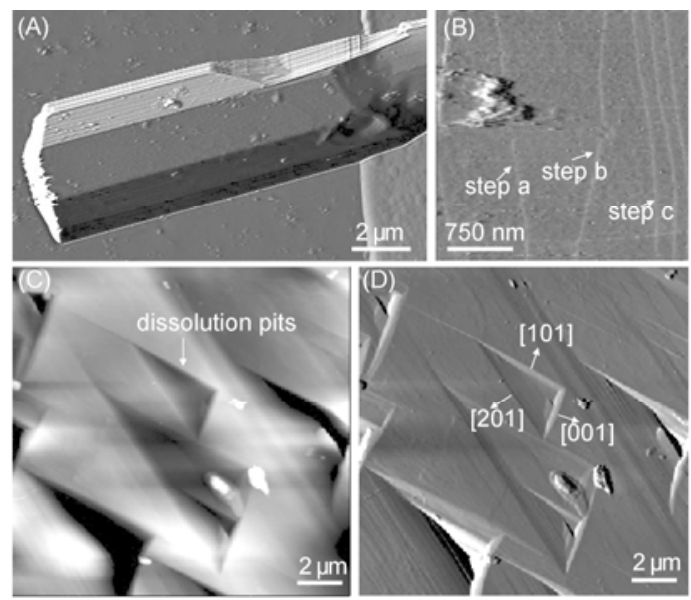

Figure 3 AFM images showing (A, B) the steps on a HAP (100) face and $(\mathrm{C}, \mathrm{D})$ the etch pits on a DCPD (010) face and their advancing directions ([001], [101], and [201]). 
[48]. Peytcheva and Antonietti [49] observed an increased dissolution rate in the presence of poly (sodium aspartate) (MW $18000 \mathrm{~g} \mathrm{~mol}^{-1}, \sim 135$ aspartic acids per molecule). They attribute the transition from shallow slow growing pits in water to faster dissolving and deeper pits in poly (sodium aspartate) to chelation of calcium and changes in the interfacial energy of the surface in the presence of the polymer. The effect of lower molecular weight poly(sodium aspartate) (MW $10000 \mathrm{~g} \mathrm{~mol}^{-1}$ ) was more like that of water, and the authors therefore argue that the enhanced pitting does not scale strictly with chelating power [49]. It should be noted that the pits are not all observed at the same degree of undersaturation (because of calcium chelation), which complicates the interpretation of the images, as some changes may be expected simply because of changes in undersaturation, independent of the effects of surfactants that alter surface energy. In particular, a higher degree of undersaturation is expected to form deeper etch pits, as the mode of dissolution changes from step flow dissolution to etch pit nucleation [37].

For an HAP (100) surface exposed to deionized water, the average height of each step was $0.84 \pm 0.1 \mathrm{~nm}$, which is close to the interlayer distance of the (100) surface $(d(100)$ $\approx 0.82 \mathrm{~nm}$ ). After exposure to citrate, surface-structuredependent asymmetric hexagonal etch pits were formed on the HAP (100) surface in which the long axis of the hexagons ran parallel to the [001] direction (Figure 4) [50]. The step energies of the $(010)$ and $(\overline{1} 10)$ facets were $4.00 \times$ $10^{-10} \mathrm{~J} \mathrm{~m}^{-1}$ and $7.68 \times 10^{-10} \mathrm{~J} \mathrm{~m}^{-1}$, respectively, suggesting that steps with (110) facets were energetically less stable than steps with (010) facets, and that the (110) face could be more susceptible to dissolution [50]. Molecular steps parallel to the elongated axes of HAP crystals, and those angled at approximately $54^{\circ}$ to the elongated axis, are not only energetically favorable but also kinetically dominant under dissolution conditions. The steps with $\theta=54^{\circ}$ can be divided into two pairs of crystallographically equivalent steps; the step velocities on one side are almost twice as fast as their counterparts on the other side, suggesting the formation of asymmetric pits [51]. Moreover, the addition of $\mathrm{NaCl}$ to the same dissolution reaction solutions $\left(10 \mathrm{mmol} \mathrm{L}^{-1}\right.$ citrate, $\mathrm{pH}$ 6) strongly suppressed HAP (100) face dissolution kinetics [52]. The step velocities and step densities were quantitatively measured, and the average dissolution rates were 3.7 $( \pm 1.7) \times 10^{-11} \mathrm{~mol} \mathrm{~m}{ }^{-2} \mathrm{~s}^{-1}, 1.7( \pm 0.6) \times 10^{-10} \mathrm{~mol} \mathrm{~m}^{-2} \mathrm{~s}^{-1}$, and $1.1( \pm 0.6) \times 10^{-9} \mathrm{~mol} \mathrm{~m}^{-2} \mathrm{~s}^{-1}$ at $1 \mathrm{~mol} \mathrm{~L}^{-1}, 100 \mathrm{mmol}$

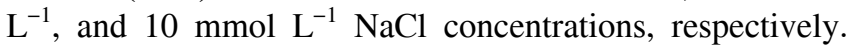
During the dissolution, the average step height on the HAP (100) face remained constant at $0.84 \pm 0.1 \mathrm{~nm}$, which corresponds to the interlayer distance between (100) surfaces. Only one type of surface termination exists in the $\mathrm{pH}$ range $6-11.7$, suggesting that the HAP surface charge is probably determined by the degree of protonation of a specific apatite surface termination rather than by interchange of a calcium or phosphate dominant surface [52]. X-ray reflectivity, which was used to determine the surface termination of HAP or fluorapatite (FAP)/water interfaces, that is, a calcium-deficient outermost layer, suggested it is the phosphate-terminated surface $[53,54]$. This result indicates that dissolution proceeds by surface protonation, which promotes the breaking of calcium-oxygen bonds. Dissolution inhibition by $\mathrm{NaCl}$ resulting from competition for surface protonation sites between $\mathrm{Na}^{+}$and $\mathrm{H}^{+}$ions is therefore proposed.

To date, most studies have just focused on the components of plant exudates that may dissolve sparingly soluble $\mathrm{CaPs}$ in soils. Almost nothing is known about the microscopic dissolution mechanisms of these CaPs in the presence of plant-root exudates such as low molecular weight organic acids, nor has the crystallization pathway from solvated ions through the intermediate phases to the least soluble CaP mineral phase been explored. The paucity of information on microscopic transformation of rhizosphere $\mathrm{P}$ in
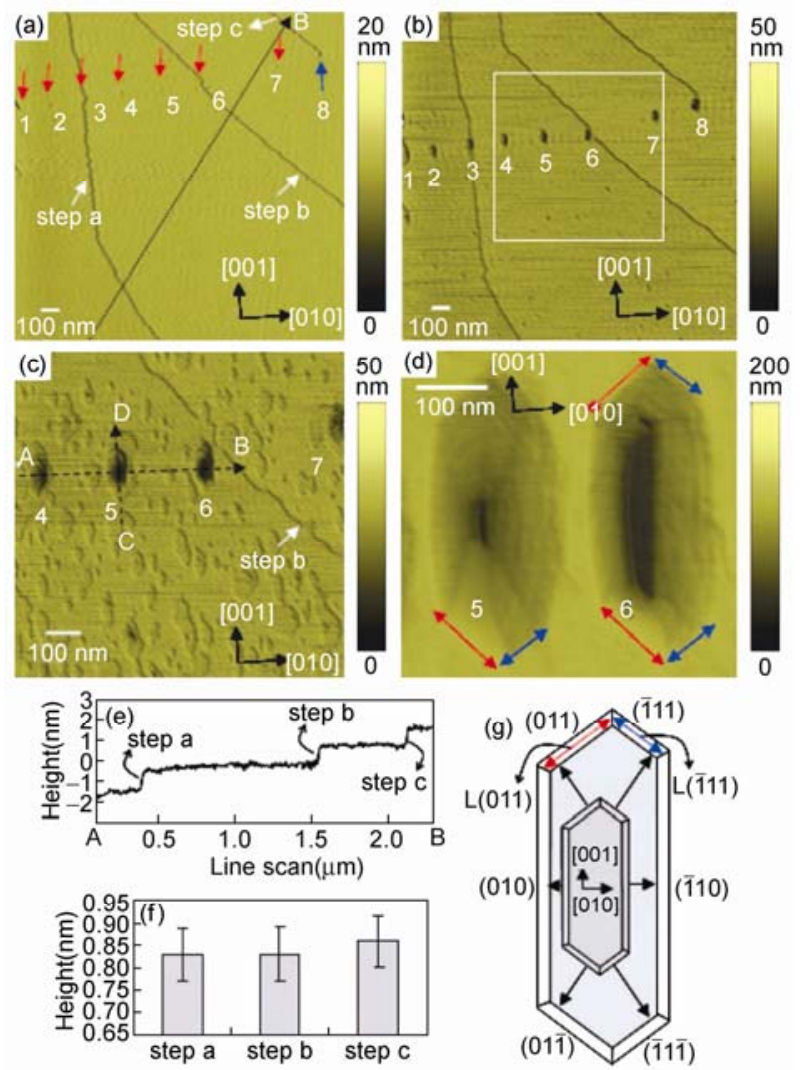

Figure 4 Structural characterization of HAP (100) surfaces during the dissolution processes. (a) AFM image of (100) surface before adding acidic buffer. White arrows indicate the step location from steps a, b, and c. Black arrows indicate edge dislocation sites (numbers from 1 to 7 ). A blue arrow indicates the screw dislocation site (number 8). (b) AFM image 5 min after injection of citrate buffer. (c) White boxed region in B (35 min later). (d) Asymmetric shape of etch pits 5 and 6 (90 min later). (e) Line scan from A to $B$ in (a). (f) Average heights of steps a-c $(0.83 \mathrm{~nm}, 0.83 \mathrm{~nm}, 0.86 \mathrm{~nm}$, respectively) from 50 measurements across each step. Error bars represent standard deviations. (g) Model of evolution of the hexagonal etch pit. Arrows represent relative step velocities displaying different facets [50]. 
different soils has been partly because of the limitations of existing experimental approaches for observation and measurement. Very recently, the cryongenic transmission electron miroscopy (cryoTEM) was employed to identify the aggregateion of prenucleation clusters that lead to the nucleationn of ACP prior to the oriented development of apatitic crystals [55]. These newly developed techniques may be applicable to understanding the complex dynamic processes of $\mathrm{CaP}$ crystallization and dissolution in geological setting (soils), the dynamics of crystallization and dissolution of $\mathrm{CaPs}$ in the presence of various influencing factors, including inorganic and organic molecules present in soil rhizosphere environments, will be investigated using in situ methods such as AFM and TEM in combination with bulk crystallization tool. The aims will be to determine the kinetics and energetics of $\mathrm{CaP}$ transformation in soil solutions or at solid surfaces at realistic driving forces, to determine the evolution of phases, to identify nucleation sites, and to define the structural relationships and stereochemical interactions that govern mineral formation or dissolution. These studies will help clarity the fundamental concepts of the biogeochemical cycle of phosphorus.

\section{Conclusion and future perspectives}

(1) During crystallization and dissolution, there are many intermediate $\mathrm{CaP}$ phases with different solubilities that suspend in soil solutions or attach to soil solid surfaces or to the ECM of soil organisms. These intermediate phases will kinetically transform into the final stable phase. Heterogeneous nucleation and growth of calcium orthophosphates from supersaturated solutions and relevant phosphate species transformations in soils have been attributed to various soluble and insoluble molecules/substrates and their combinations with proteins, peptides, enzymes, and inorganic ions, on the assumption that each exhibits roughly the same influence in promoting or inhibiting the earliest nucleation and subsequent growth of CaP crystallites. (2) Dissolution reactions of $\mathrm{CaPs}$ may be inhibited or even suppressed when the crystallite sizes fall into the critical nanoscale range. This phenomenon involves particle-size-dependent critical conditions of energetic control at the molecular level. The formation of etch pits on the crystal faces is the driving force for dissolution. It has been shown that only pits which are larger than the critical size provide active dissolution sites that contribute to dissolution.

Based on progress in research on $\mathrm{CaP}$ biomineralization and the relevant solution chemistry, in combination with AFM studies of crystal surface dynamics, studies of the phosphates in soils and their transformation at the near-molecular level will be possible. There are two possible directions. (1) A combination of solution chemistry, in situ AFM, and molecular modeling, will enable the parameters of the kinetics and thermodynamics of the initial nucleation, phase stability, and subsequent growth of CaPs in solutions or on solid substrates such as Fe/Al oxides and ECM of bacteria to be determined; the structural relationships and stereochemical interactions that govern mineral formation on substrates may also be defined. (2) Dissolution processes of sparingly soluble CaPs in the presence of various possible plant-root exudates such as small organic acids will be observed by in situ AFM to show pit densities and surface geometries, and size and step spreading velocities quantitatively. These microscopic studies can explain the bulk dissolution of sparingly soluble $\mathrm{CaP}$ salts, which is generally initiated and accompanied by the formation and development of pits on the mineral surfaces. These studies may enable the fundamental concepts of phosphate use in soils to be clarified and understood.

This work was supported by the National Natural Science Foundation of China (41071208) and a start-up grant from the Huazhong Agricultural University (52204-09008). We thank Dr. Duanwei Zhu for constructive discussions and suggestions.

1 Filippelli G M. The global phosphorous cycle: Past, present, and future. Elements, 2008, 4: 89-95

2 Gilbert N. The disappearing nutrient. Nature, 2009, 461: 716-718

3 Raghothama K G. Phosphate acquisition. Ann Rev Plant Physiol Plant Mol Biol, 1999, 50: 665-693

4 Rosling A, Suttle K B, Johansson E, et al. Phosphorous availability influences the dissolution of apatite by soil Fungi. Geobiology, 2007, 5: 265-280

5 Manning D A C. Phosphate minerals, environmental pollution and sustainable agriculture. Elements, 2008, 4: 105-108

6 Wang L J, Nancollas G H. Calcium orthophosphates: Crystallization and dissolution. Chem Rev, 2008, 108: 4628-4669

7 Wang L J, Nancollas G H. Pathways to biomineralization and biodemineralization of calcium phosphates: The thermodynamic and kinetic controls. Dalton Trans, 2009, 2665-2672

8 Chernov A A. Notes on interface growth kinetics 50 years after Burton, Cabrera and Frank. J Cryst Growth, 2004, 264: 499-518

9 Zhang J W, Nancollas G H. Kink density and rate of step movement during growth and dissolution of an $\mathrm{AB}$ crystal in a nonstoichiometric solution. J Colloid Interface Sci, 1998, 200: 131-145

10 Cuppen H M, Meekes H, van Enckevort W J P, et al. Birth-andspread growth on the Kossel and a non-Kossel surface. J Cryst Growth, 2006, 286: 188-196

11 Chernov A A, Petrova E, Rashkovich L N. Dependence of the $\mathrm{CaO}_{x}$ and $\mathrm{MgO}_{x}$ growth rate on solution stoichiometry. Non-Kossel crystal growth. J Cryst Growth, 2006, 289: 245-254

12 Politi Y, Batchelor D R, Zaslansky P, et al. Role of magnesium ion in the stabilization of biogenic amorphous calcium carbonate: A structure-function investigation. Chem Mater, 2010, 22: 161-166

13 Wang L J, Nancollas G H. In Biomineralization. From nature to application; Vol. 4. In: Sigel A, Sigel H, Sigel R K O, eds. Metal Ions in Life Sciences. Chichester: John Wiley \& Sons, 2008. 413

14 Fuierer T A, LoRe M, Puckett S A, et al. A mineralization adsorption and mobility study of hydroxyapatite surfaces in the presence of zinc and magnesium ions. Langmuir, 1994, 10: 4721-4725

15 Wang L J, Guan X Y, Du C, et al. Amelogenin promotes the formation of elongated apatite microstructures in a controlled crystallization system. J Phys Chem C, 2007, 111: 6398-6404

16 Wang L J, Guan X Y, Yin H Y, et al. Mimicking the self-organized microstructure of tooth enamel. J Phys Chem C, 2008, 112: 58925899 
17 Yang X D, Wang L J, Qin Y L, et al. How amelogenin orchestrates the organization of hierarchical elongated microstructures of apatite. $\mathrm{J}$ Phys Chem B, 2010, 114: 2293-2300

18 Ikawa N, Kimura T, Oumi Y, et al. Amino acid containing amorphous calcium phosphates and the rapid transformation into apatite. $\mathrm{J}$ Mater Chem, 2009, 19: 4906-4913

19 Wang L J, Guan X Y, Tang R K, et al. Phosphorylation of osteopontin is required for inhibition of calcium oxalate crystallization. J Phys Chem B, 2008, 112: 9151-9157

20 Guan X Y, Wang L J, Dosen A, et al. An understanding of renal stone development in a mixed oxalate-phosphate system. Langmuir, 2008, 24: 7058-7060

21 Wang L J, De Yoreo J J, Guan X Y, et al. Constant composition studies verify the utility of the Cabrera-Vermilyea $(\mathrm{C}-\mathrm{V})$ model in explaining mechanisms of calcium oxalate monohydrate crystallization. Cryst Growth Des, 2006, 6: 1769-1775

22 Wang L J, Qiu S R, Zachowicz W Z, et al. Modulation of calcium oxalate crystallization by linear aspartic acid-rich peptides. Langmuir, 2006, 22: 7279-7285

23 Wang L J, Zhang W, Qiu S R, et al. Inhibition of calcium oxalate monohydrate crystallization by the combination of citrate and osteopontin. J Cryst Growth, 2006, 291: 160-165

24 Bar-Yosef Ofir P, Govrin-Lippman R, Garti N, et al. The influence of polyelectrolytes on the formation and phase transformation of amorphous calcium phosphate. Cryst Growth Des, 2004, 4: 177-183

25 Jiang W, Chu X, Wang B, et al. Biomimetically triggered inorganic crystal transformation by biomolecules: A new understanding of biomineralization. J Phys Chem B, 2009, 113: 10838-10844

26 Dorozhkin S V, Epple M. Biological and medical significance of calcium phosphates. Angew Chem Int Ed, 2002, 41: 3130-3146

27 Zhang Z, Ma G, Liu X Y. Will fluoride toughen or weaken our teeth? Understandings based on nucleation, morphology, and structural assembly. J Phys Chem B, 2009, 113: 16393-16399

28 Land T A, De Yoreo J J, Lee J D. An in situ AFM investigation of canavalin crystallization kinetics. Surf Sci, 1997, 384: 136-155

29 Teng H H, Dove P M, Orme C A, et al. The thermodynamics of calcite growth: A baseline for biomineralization. Science, 1998, 282: 724-727

30 Pina C M, Becker U, Risthaus P, et al. Molecular-scale mechanisms of crystal growth in barite. Nature, 1998, 395: 483-486

31 Chernov A A. Modern Crystallography III: Crystal Growth. Berlin: Springer-Verlag, 1984

32 De Yoreo J J, Zepeda-Ruiz L A, Friddle R W, et al. Rethinking classical crystal growth models through molecular scale insights: Consequences of kink-limited kinetics. Cryst Growth Des, 2009, 9: 5135-5144

33 Land T A, Martin T L, Potapenko S, et al. Recovery of surfaces from impurity poisoning during crystal growth. Nature, 1999, 399: 442445

34 Davis K J, Dove P M, De Yoreo J J. The role of $\mathrm{Mg}^{2+}$ as an impurity in calcite growth. Science, 2000, 290: 1134-1137

35 De Yoreo J J, Vekilov P G. Reviews in Mineralogy and Geochemistry 54. Washington, DC: Mineralogical Society of America, 2003. 57-93

36 Orme C A, Giocondi J L. In: Skowronski M, De Yoreo J J, Wang C
A, eds. Perspectives on Inorganic, Organic and Biological Crystal Growth. AIP Conference Proceeding, Melville, 2007. Vol. 916

37 Qiu S R, Orme C A. Dynamics of biomineral formation at the near-molecular level. Chem Rev, 2008, 108: 4784-4822

38 Tang R K, Nancollas G H, Orme C A. Mechanism of dissolution of sparingly soluble electrolytes. J Am Chem Soc, 2001, 123: 54375443

39 Tang R K, Orme C A, Nancollas G H. A new understanding of demineralization: The dynamics of brushite dissolution. J Phys Chem B, 2003, 107: 10653-10657

40 Tang R K, Wang L J, Orme C A, et al. Dissolution at the nanoscale: Self-preservation of biominerals. Angew Chem Int Ed, 2004, 43: 2697-2701

41 Tang R K, Wang L J, Nancollas G H. Size-effects in the dissolution of hydroxyapatite: An understanding of biological demineralization. J Mater Chem, 2004, 14: 2341-2346

42 Wang L J, Tang R K, Bonstein T, et al. A new model for nanoscale enamel dissolution. J Phys Chem B, 2005, 109: 999-1005

43 Wang L J, Tang R K, Bonstein T, et al. Enamel demineralization in primary and permanent teeth. J Dent Res, 2006, 85: 359-363

44 Wang L J, Nancollas G H, Henneman Z, et al. Nanosized particles in bone and dissolution insensitivity of bone mineral. Biointerphases, 2006, 1: 106-111

45 Qiu S R, Wierzbicki A, Orme C A, et al. Molecular modulation of calcium oxalate crystallization by osteopontin and citrate. Proc Natl Acad Sci USA, 2004, 101: 1811-1815

46 Tang R K, Darragh M, Orme C A, et al. Control of biomineralization dynamics by interfacial energies. Angew Chem Int Ed, 2005, 44: 3698-3702

47 Filgueiras M R T, Mkhonto D, De Leeuw N H. Computer simulations of the adsorption of citric acid at hydroxyapatite surfaces. J Cryst Growth, 2006, 294: 60-68

48 Jiang W, Pan H H, Cai Y R, et al. Atomic force microscopy reveals hydroxyapatite-citrate interfacial structure at the atomic level. Langmuir, 2008, 24: 12446-12451

49 Peytcheva A, Antonietti M. Carving on the nanoscale: Polymers for the site-specific dissolution of calcium phosphate. Angew Chem Int Ed, 2001, 40: 3380-3383

50 Kwon K Y, Wang E, Chung A, et al. Defect induced asymmetric pit formation on hydroxyapatite. Langmuir, 2008, 24: 11063-11066

51 Kwon K Y, Wang E, Chang N, et al. Characterization of the dominant molecular step orientations on hydroxyapatite (100) surfaces. Langmuir, 2009, 25: 7205-7208

52 Kwon K Y, Wang E, Chung A, et al. Effect of salinity on hydroxyapatite dissolution studied by atomic force microscopy. J Phys Chem C, 2009, 113: 3369-3372

53 Park C Y, Fenter P, Zhang Z, et al. Structure of the fluorapatite (100)-water interface by high-resolution X-ray reflectivity. Am Mineral, 2004, 89: 1647-1654

54 Pareek A, Torrelles X, Rius J, et al. Role of water in the surface relaxation of the fluorapatite (100) surface by grazing incidence X-ray diffraction. Phys Rev B, 2007, 75: 035418

55 Dey A, Bomans P H H, Müller F A, et al. The role of prenuclation clusters in surface-induced calcium phosphate crystallization. Nat Mater, 2010, 9: 1010-1014

Open Access This article is distributed under the terms of the Creative Commons Attribution License which permits any use, distribution, and reproduction in any medium, provided the original author(s) and source are credited. 